\title{
INSTRUMENTOS DE AVALIAÇÃO BREVE DA COMUNICAÇÃO: FERRAMENTAS EXISTENTES E SUA APLICABILIDADE CLÍNICA
}

\author{
Brief communication assessment instruments: \\ available tools and their clinical applicability
}

\author{
Fabíola Schwengber Casarin ${ }^{(1)}$, Karina Carlesso Pagliarin ${ }^{(2)}$, Cristine Koehler ${ }^{(3)}$, \\ Camila Rosa de Oliveira $^{(4)}$, Rochele Paz Fonseca ${ }^{(5)}$
}

\begin{abstract}
RESUMO
Tema: a avaliação breve da comunicação é um recurso útil aos profissionais da saúde, pois fornece informações que subsidiam tanto o diagnóstico funcional, quanto o planejamento e a execução de avaliações expandidas e de medidas de reabilitação a serem realizados em cada caso. Objetivo: apresentar uma revisão sistemática acerca do uso de instrumentos padronizados de avaliação breve da comunicação e caracterizar as populações alvo descritas. Conclusão: encontraram-se 18 artigos, com 10 instrumentos breves, sendo a população mais examinada a de pacientes pós-acidente vascular cerebral. Os componentes lingüísticos prevalentes nas avaliações foram expressão, compreensão, leitura e escrita.
\end{abstract}

DESCRITORES: Avaliação; Linguagem; Comunicação

\section{INTRODUÇÃO}

Essa revisão sistemática aborda uma temática de interface entre a Fonoaudiologia e a Neuropsicologia: a avaliação breve da comunicação. O exame do processamento comunicativo em pacientes adultos tem sido amplamente utilizada na prática

(1) Fonoaudióloga; Mestranda em Psicologia pela Pontifícia Universidade Católica do Rio Grande do Sul, PUCRS, Porto Alegre, RS, Brasil; Bolsista Capes.

(2) Fonoaudióloga; Doutoranda em Psicologia pela Pontifícia Universidade Católica do Rio Grande do Sul PUCRS, Porto Alegre, RS, Brasil; Bolsista CNPq.

(3) Acadêmica do Curso de Fonoaudióloga da Universidade Federal de Ciências da Saúde de Porto Alegre- UFCSPA, Porto Alegre, RS, Brasil.

(4) Psicóloga; Mestranda em Psicologia pela Pontifícia Universidade Católica do Rio Grande do Sul PUCRS, Porto Alegre, RS, Brasil; Bolsista CNPq.

(5) Psicóloga e Fonoaudióloga; Professora do Curso de Psicologia e o Programa de Pós-graduação em Psicologia, área de concentração Cognição Humana, da Pontifícia Universidade Católica do Rio Grande do Sul PUCRS, Porto Alegre, RS, Brasil; Doutora em Psicologia pela Universidade Federal do Rio Grande do Sul; Pós-Doutoranda em Ciências Biomédicas, Centro de Neuroimagem pela Universidade de Montreal.

Conflito de interesses: inexistente clínica fonoaudiológica e neuropsicológica, principalmente, em distúrbios neurológicos decorrentes de acidente vascular cerebral (AVC) ${ }^{1}$, traumatismo crânio-encefálico (TCE) ${ }^{2}$, doenças neurológicas degenerativas, como a demência ${ }^{3}$. Inúmeros instrumentos são descritos na literatura para avaliar as habilidades linguísticas e comunicativas dos indivíduos com algum comprometimento neurológico. Entre os mais citados estão Aphasia Diagnostic Profiles ${ }^{4}$, Boston Assessment of Severe Aphasia (BASA) ${ }^{5}$, Boston Diagnostic Aphasia Examination (BDAE) ${ }^{6}$, Boston Naming Test (BNT) ${ }^{7}$, Minnesota Test for Differential Diagnosis of Aphasia (MTDDA), Multilingual Aphasia Examination (MAE) ${ }^{8}$, Neurosensory Center Comprehensive Examination for Aphasia (NCCEA) ${ }^{9}$, Porch Index Communicative Ability (PICA) ${ }^{10}$, Reading Comprehension Battery for Aphasia (RCBA) ${ }^{11}$, Token Test ${ }^{12}$ e Western Aphasia Battery ${ }^{13}$. Entretanto, em geral, tais ferramentas clínicas requerem um número de sessões que varia de duas a oito, aproximadamente, tempo de avaliação não condizente com a realidade dos serviços de saúde prestados no Brasil e, até mesmo, na América Latina ${ }^{14}$.

Pelo fato de que a realidade da saúde pública brasileira é caracterizada por uma demanda de avaliações clínicas em múltiplas especialidades de 
rápida aplicação e interpretação, torna-se importante o uso de baterias breves de avaliação cognitiva realizada rotineiramente como parte do exame neurológico ${ }^{15}$. Além disso, a investigação breve da comunicação pode ser um instrumento útil na avaliação global do paciente, permitindo aos neurologistas, psiquiatras, geriatras, fonoaudiólogos ou neuropsicólogos obterem informações que subsidiem tanto o diagnóstico funcional do quadro em questão quanto o planejamento e a execução do aprofundamento da avaliação e da reabilitação cognitiva e comunicativa a serem realizados em cada caso ${ }^{16}$.

Em face desta demanda, é crescente o número de estudos de desenvolvimento de baterias breves que avaliam o perfil cognitivo de uma ou mais funções neuropsicológicas. Pode-se citar, por exemplo, o NEUROPSI ${ }^{14}$, na língua espanhola, e o NEUPSILIN ${ }^{17}$, no idioma Português Brasileiro, os quais avaliam diversas funções cognitivas. Nos testes de inteligência também se encontram versões breves de mensuração como a Wechsler Abbreviated Scale of Intelligence - WASI (Wechsler) ${ }^{18}$. Já outros testes breves visam a avaliar habilidades específicas, como a Frontal Assessment Battery - FAB (Dubois, Slachevsky, Litvan \& Pillon) ${ }^{19}$ constituído de seis subtestes que mensuram, em aproximadamente 10 minutos, componentes executivos como a conceitualização, a flexibilidade mental, a programação motora, a sensibilidade para inferências, o controle inibitório e a autonomia ambiental. Outro exemplo é o Teste Wisconsin de Classificação de Cartas (WCST), versão reduzida de 48 cartões ${ }^{20}$, que também se destina a fornecer medidas de componentes das funções executivas, tais como planejamento, abstração, aprendizagem, manutenção de regras bem-sucedidas e flexibilidade cognitiva. No entanto, a avaliação de habilidades comunicativas, tanto no contexto nacional quanto internacional, ainda carece de instrumentos breves.

Dessa forma, esse estudo teve como objetivo apresentar uma revisão sistemática acerca do uso de instrumentos padronizados de avaliação breve da comunicação e caracterizar as populações alvo descritas. A partir dessa análise, pretendese responder às seguintes questões de pesquisa: 1) Quais os instrumentos breves utilizados na avaliação da comunicação em adultos? 2) Quais as populações examinadas nesses estudos? 3) Quais os componentes comunicativos mais avaliados por esses instrumentos?

\section{MÉTODO}

Em junho de 2010, realizou-se uma busca sistemática da literatura por meio de uma pesquisa nas bases de dados internacionais PubMed, LILACS e HaPI, incluindo abstracts de artigos publicados de 1985 a 2010. Este intervalo inclui o período inicial em que houve um aumento expressivo do desenvolvimento de ferramentas padronizadas de avaliação de componentes linguísticos funcionais ${ }^{21}$ e estende-se até o ano atual.

Foram utilizadas as seguintes associações de palavras-chave: construto avaliação AND construto comunicação AND construto população examinada. Para cada construto as palavras-chave utilizadas foram as que seguem: a) construto avaliação: "brief assessment" (avaliação breve) OR "brief evaluation" OR "short assessment" OR "short evaluation" (avaliação curta) OR "short battery" (bateria curta) OR "short version" (versão reduzida) OR "short form" (versão pequena) OR "rapidly administered" (adminstração rápida) OR "screening"; b) construto comunicação, representado por termos dos mais amplos aos mais específicos, considerando-se os níveis da palavra, da sentença e do discurso, além dos diferentes componentes linguísticos: "communication" (comunicação) OR "language" (linguagem) OR "discourse" (discurso) OR "pragmatics" (pragmática) OR "prosody" (prosódia) OR "semantics" (semântica) OR "syntax" (sintaxe); C) construto população examinada: "clinical population" (população clínica) ou "aphasia" (afasia) OR "brain injury" (lesão cerebral) OR "closed head injury" (TCE fechado) OR "stroke" (AVC). Tais palavras-chave relacionadas a quadros clínicos foram selecionadas frente à alta prevalência de sequelas comunicativas após sua ocorrência ${ }^{22}$. Todos os termos escolhidos estão catalogados pela base de termos Medical Subjects Headings (MeSH), exceto "clinical population", que foi mantida devido à quantidade de estudos que consideram esta variável.

A primeira busca de resumos gerou uma quantificação inicial de abstracts a serem analisados. Desses abstracts, foram incluídos na quantificação final apenas aqueles que cumpriram os critérios: a) ter como objeto de estudo empírico um instrumento padronizado de avaliação breve da comunicação, b) incluir na sua amostra apenas indivíduos adultos, c) ser publicado nos idiomas inglês, francês, espanhol ou português. Os abstracts repetidos foram excluídos, ou seja, foram contabilizados apenas uma vez. Todos os artigos completos referentes à busca final foram, então, analisados na íntegra para responder às questões de pesquisa, sendo os resultados analisados descritivamente. 
Em complementaridade, fez-se uma pesquisa em uma base de dados específica, a Health and Psychosocial Instruments Database (HaPl), mediante consultoria especializada de duas bibliotecárias da Universidade de Montreal (Institut Universitaire de Gériatrie de Montreal - IUGM). Esta base fundada em 1985 fornece acesso rápido de instrumentos de medidas aos profissionais do campo da saúde, da ciência psicossocial, da organização comportamental, das bibliotecas e da ciência de informação. Está baseada em termos oriundos de linguagem natural, ou seja, conforme o conhecimento dos autores obtido pelo largo contato com a literatura específica. Por ser uma base de informações sobre ferramentas de avaliação, utilizou-se uma sintaxe com os seguintes termos dos construtos avaliação (todos com OR), evaluation, assessment, test, short, rapidly, screening and brief, AND comunicação (todos com OR), communication, language, discourse, pragamatic*, semantic*, prodody ${ }^{*}$, syntax, narrative, conversation. Para as palavras do construto avaliação, foram selecionados os campos de pesquisa, ti (title), so (source) e if (references), e para o construto comunicação, md (measure descriptors), sd (sample descriptors) e de (descriptors).

\section{REVISÃO DA LITERATURA}

O resultado da busca de resumos que cumpriram todos os critérios de inclusão descritos na seção anterior podem ser visualizados em duas tabelas. A Tabela 1 apresenta o número total de abstracts encontrados e analisados nas bases de dados Pubmed, HaPI e LILACS, e o número de artigos selecionados após refinamento, conforme os descritores escolhidos e especificados na Seção Método.

Tabela 1 - Quantidade de abstracts selecionados pós-análise de critérios de inclusão

\begin{tabular}{cccc}
\hline Base de dados & $\mathbf{N}^{\circ}$ inicial de abstracts & $\begin{array}{c}\mathbf{N}^{\circ} \text { artigos } \\
\text { analisados }\end{array}$ & $\begin{array}{c}\mathbf{N}^{\circ} \text { artigos } \\
\text { selecionados }\end{array}$ \\
\hline Pubmed & 885 & 36 & 12 \\
HaPI & 1078 & 19 & 6 \\
LILACS & 6 & 0 & 0 \\
\hline
\end{tabular}

Foram encontrados 18 artigos, todos em bases internacionais. A Tabela 2 apresenta os instrumentos de avaliação breve da comunicação e os screenings selecionados, com os seus respectivos autores, amostra, tempo de administração e componentes comunicativos avaliados.

Os 18 estudos utilizaram variadas baterias breves e screenings de avaliação da comunicação. A partir da Tabela 2, é possível observar que o Frenchay Aphasia Screening Test (FAST), além de ser o teste mais antigo, foi também o mais utilizado na maioria dos estudos (sete) analisados. O tempo máximo de administração destes instrumentos foi de 45 minutos.

No que diz respeito à primeira questão de pesquisa dessa revisão sistemática sobre quais instrumentos de avaliação da comunicação breve são utilizados para a população adulta, foram encontrados dez instrumentos. Durante o processo de análise verificou-se que os testes relatados nos artigos diferenciam entre screenings ${ }^{23-35,27,31,33-40} \mathrm{e}$ avaliações breves 26,28-30,32.

Embora não haja, até onde se sabe, uma classificação consensual na literatura dos instrumentos neuropsicológicos quanto ao tempo de administração, sabe-se que os screenings possuem uma aplicação mais rápida, entre 3 a 15 minutos, e determinam ausência ou presença de um déficit neuropsicológico, neste caso de linguagem, e auxiliam o encaminhamento de avaliações aprofundadas e planejamento inicial de reabilitação, quando necessárias ${ }^{41}$, não oferecendo diagnóstico. Já nas avaliações breves, o tempo de aplicação é maior, podendo durar até 45 minutos, equivalente a uma sessão de avaliação, com uma maior variedade de funções cognitivas examinadas, e contribuindo de modo mais aprofundado que os screenings para o processo diagnóstico, prognóstico e de tratamento. Cada subteste consiste da observação e descrição do comportamento do paciente quando confrontado pela demanda cognitiva da tarefa. É a partir dessas observações e medidas que o clínico pode fazer uma avaliação formal da habilidade em questão. A avaliação breve é indicada para um panorama inicial da função cognitiva examinada sendo essencial para a seleção dos próximos recursos de avaliação, ou quando não se dispõe de tempo suficiente para a realização de uma bateria completa ou, ainda, quando o indivíduo apresenta um tempo curto de atenção concentrada e/ou de consciência, como no caso de pacientes hospitalizados e/ou que sofreram alguma lesão cerebral severa ${ }^{42}$. 
Tabela 2 - Instrumentos de avaliação breve e screenings

\begin{tabular}{|c|c|c|c|c|c|}
\hline \multirow[b]{2}{*}{ Autores } & \multicolumn{5}{|c|}{ Método } \\
\hline & $\begin{array}{l}\text { População } \\
\text { clínica }\end{array}$ & Controles & $\begin{array}{l}\text { Instrumentos } \\
\text { breves }\end{array}$ & $\begin{array}{c}\text { Tempo de } \\
\text { administração }\end{array}$ & $\begin{array}{c}\text { Componentes } \\
\text { comunicativos } \\
\text { avaliados }\end{array}$ \\
\hline $\begin{array}{l}\text { 1. Hilari et al. } \\
(2010)^{23}\end{array}$ & $\begin{array}{l}71 \text { pacientes } \\
\text { com AVC }\end{array}$ & - & $\begin{array}{l}\text { Frenchay } \\
\text { Aphasia } \\
\text { Screening } \\
\text { Test (FAST) }\end{array}$ & 3-10 minutos & $\begin{array}{l}\text { Compreensão, } \\
\text { expressão, } \\
\text { leitura e } \\
\text { escrita }\end{array}$ \\
\hline $\begin{array}{l}\text { 2. Hilari et al. } \\
(2009)^{24}\end{array}$ & $\begin{array}{l}126 \text { pacientes } \\
\text { com AVC }\end{array}$ & - & $\begin{array}{l}\text { Frenchay } \\
\text { Aphasia } \\
\text { Screening } \\
\text { Test (FAST) } \\
\text { Short Form of } \\
\text { the Boston } \\
\text { Naming Test } \\
\text { (BNT) }\end{array}$ & - & $\begin{array}{l}\text { Compreensão, } \\
\text { expressão, } \\
\text { leitura e } \\
\text { escrita }\end{array}$ \\
\hline $\begin{array}{l}\text { 3. Long, Hesketh } \\
\text { \& Bowen (2009) } \\
25\end{array}$ & $\begin{array}{l}58 \\
\text { sobreviventes } \\
\text { de AVC com } \\
\text { problemas de } \\
\text { comunicação } \\
\text { (afasia e/ou } \\
\text { disartria) }\end{array}$ & - & $\begin{array}{l}\text { Frenchay } \\
\text { Aphasia } \\
\text { Screening } \\
\text { Test (FAST) }\end{array}$ & 3-10 minutos & $\begin{array}{l}\text { Compreensão, } \\
\text { expressão, } \\
\text { leitura e } \\
\text { escrita }\end{array}$ \\
\hline $\begin{array}{l}\text { 4. Casas, } \\
\text { Calamia \& Tranel } \\
(2008)^{26}\end{array}$ & - & $\begin{array}{l}67 \text { adultos } \\
\text { bilíngues }\end{array}$ & $\begin{array}{l}\text { Teste breve } \\
\text { de avaliação } \\
\text { das } \\
\text { dificuldades } \\
\text { de nomeação } \\
\text { visual em } \\
\text { bilíngues }\end{array}$ & - & Nomeação \\
\hline $\begin{array}{l}\text { 5. Kostalova et } \\
\text { al. }(2008)^{27}\end{array}$ & $\begin{array}{l}149 \text { pacientes } \\
\text { com AVC } \\
\text { isquêmico ou } \\
\text { hemorrágico } \\
\text { unilateral } \\
\text { esquerdo }\end{array}$ & $\begin{array}{l}\text { Grupo clínico: } \\
45 \text { pacientes } \\
\text { com AVC } \\
\text { isquêmico ou } \\
\text { hemorrágico } \\
\text { unilateral } \\
\text { direito sem } \\
\text { afasia. } \\
\text { Grupo não } \\
\text { clínico: } 243 \\
\text { participantes } \\
\text { saudáveis }\end{array}$ & $\begin{array}{l}\text { Mississippi } \\
\text { Aphasia } \\
\text { Screening } \\
\text { Test (MAST) }\end{array}$ & 5-10 minutos & $\begin{array}{l}\text { Nomeação, } \\
\text { fala } \\
\text { automática, } \\
\text { repetição, } \\
\text { resposta } \\
\text { sim/não, } \\
\text { reconheciment } \\
\text { o de objetos } \\
\text { entre cinco } \\
\text { opções, seguir } \\
\text { instruções, ler } \\
\text { instruções, } \\
\text { fluência } \\
\text { verbal, ditado, } \\
\text { escrita/soletra } \\
\text { ção }\end{array}$ \\
\hline $\begin{array}{l}\text { 6. Laska, Kahan, } \\
\text { Hellblom, Murray } \\
\text { \& von Arbin } \\
(2008)^{28}\end{array}$ & $\begin{array}{l}41 \text { pacientes } \\
\text { com AVC } \\
\text { isquêmico } \\
\text { agudo }\end{array}$ & $\begin{array}{l}38 \text { pacientes } \\
\text { com AVC } \\
\text { isquêmico } \\
\text { agudo }\end{array}$ & $\begin{array}{l}\text { Short form of } \\
\text { the Norsk } \\
\text { Grunntest for } \\
\text { Aphasia } \\
\text { (NGA) }\end{array}$ & $\begin{array}{c}15 \\
\text { minutos }\end{array}$ & $\begin{array}{l}\text { Fluência } \\
\text { verbal, } \\
\text { compreensão, } \\
\text { nomeação, } \\
\text { repetição, } \\
\text { leitura e } \\
\text { escrita }\end{array}$ \\
\hline
\end{tabular}




\begin{tabular}{|c|c|c|c|c|c|}
\hline $\begin{array}{l}\text { 7. Sabe et al. } \\
(2008)^{29}\end{array}$ & $\begin{array}{l}121 \text { pacientes } \\
\text { com lesão } \\
\text { cerebral } \\
\text { afásicos }\end{array}$ & $\begin{array}{l}77 \text { pacientes } \\
\text { com lesão } \\
\text { cerebral } \\
\text { disártricos } \\
\text { sem patologia } \\
\text { linguística } \\
\text { associada }\end{array}$ & $\begin{array}{l}\text { Bedside Del } \\
\text { Languaje - BL }\end{array}$ & $<15$ minutos & $\begin{array}{l}\text { Linguagem } \\
\text { espontânea, } \\
\text { compreensão, } \\
\text { repetição, } \\
\text { escrita e } \\
\text { leitura }\end{array}$ \\
\hline $\begin{array}{l}\text { 8. Theodoros et } \\
\text { al. }(2008)^{30}\end{array}$ & $\begin{array}{l}32 \text { pacientes } \\
\text { ( } 31 \text { com AVC } \\
\text { e } 1 \text { com TCE) }\end{array}$ & - & $\begin{array}{l}\text { Short Form of } \\
\text { the Boston } \\
\text { Diagnostic } \\
\text { Aphasia } \\
\text { Examination, } \\
\text { 3rd edition } \\
\text { (BDAE-3) } \\
\text { Short Form of } \\
\text { the Boston } \\
\text { Naming Test } \\
\text { (BNT) }\end{array}$ & $30-45$ minutos & $\begin{array}{l}\text { Linguagem } \\
\text { espontânea, } \\
\text { nomeação, } \\
\text { compreensão, } \\
\text { repetição, } \\
\text { escrita e } \\
\text { leitura }\end{array}$ \\
\hline $\begin{array}{l}\text { 9. Edwards et al. } \\
(2006)^{31}\end{array}$ & $\begin{array}{l}53 \text { pacientes } \\
\text { com AVC } \\
\text { agudo, média } \\
\text { de idade de } 64 \\
\text { anos }\end{array}$ & - & $\begin{array}{l}\text { Frenchay } \\
\text { Aphasia } \\
\text { Screening } \\
\text { Test (FAST) }\end{array}$ & 3-10 minutos & $\begin{array}{l}\text { Compreensão, } \\
\text { expressão, } \\
\text { leitura e } \\
\text { escrita }\end{array}$ \\
\hline $\begin{array}{l}\text { 10. Kalbe, } \\
\text { Reinhold, Brand, } \\
\text { Markowitsch \& } \\
\text { Kessler (2005) }\end{array}$ & $\begin{array}{l}154 \text { pacientes } \\
\text { afásicos }\end{array}$ & $\begin{array}{l}106 \\
\text { participantes } \\
\text { saudáveis }\end{array}$ & $\begin{array}{l}\text { Aphasia } \\
\text { Check List } \\
\text { (ACL) }\end{array}$ & $\begin{array}{c}30 \\
\text { minutos }\end{array}$ & $\begin{array}{l}\text { Compreensão } \\
\text { e expressão } \\
\text { da linguagem, } \\
\text { leitura, escrita, } \\
\text { repetição, } \\
\text { linguagem } \\
\text { automática, } \\
\text { compreensão } \\
\text { de instruções } \\
\text { verbais, } \\
\text { compreensão } \\
\text { auditiva, } \\
\text { material verbal } \\
\text { complexo e } \\
\text { tarefas de } \\
\text { geração de } \\
\text { palavras }\end{array}$ \\
\hline $\begin{array}{l}\text { 11. Nakase- } \\
\text { Thompson et al. } \\
(2005)^{33}\end{array}$ & $\begin{array}{l}38 \text { pacientes } \\
\text { com Lesão de } \\
\text { hemisférios } \\
\text { esquerdo, e } \\
20 \text { pacientes } \\
\text { com lesão de } \\
\text { hemisférios } \\
\text { direito }\end{array}$ & $\begin{array}{l}6 \\
\text { participantes } \\
\text { saudáveis }\end{array}$ & $\begin{array}{l}\text { Mississippi } \\
\text { Aphasia } \\
\text { Screening } \\
\text { Test (MAST) }\end{array}$ & 5-10 minutos & $\begin{array}{l}\text { Nomeação, } \\
\text { linguagem } \\
\text { automática, } \\
\text { repetição, } \\
\text { perguntas } \\
\text { fechadas, } \\
\text { ditado, } \\
\text { reconheciment } \\
\text { o de objeto, } \\
\text { compreensão } \\
\text { de instruções } \\
\text { verbais e } \\
\text { escritas, } \\
\text { fluência } \\
\text { verbal, índice } \\
\text { expressivo e } \\
\text { receptivo }\end{array}$ \\
\hline
\end{tabular}




\begin{tabular}{|c|c|c|c|c|c|}
\hline $\begin{array}{l}\text { 12. Blake, } \\
\text { McKinney, } \\
\text { Treece, Lee \& } \\
\text { Linconl (2002) }\end{array}$ & $\begin{array}{l}112 \text { Pacientes } \\
\text { pós AVC }\end{array}$ & & $\begin{array}{l}\text { Sheffield } \\
\text { Screening } \\
\text { Test }\end{array}$ & - & $\begin{array}{l}\text { Tarefas de } \\
\text { compreensão } \\
\text { e produção }\end{array}$ \\
\hline $\begin{array}{l}\text { 13. Thommessen, } \\
\text { Thoresen, Bautz- } \\
\text { Holter, \& Laake } \\
(1999){ }^{35}\end{array}$ & $\begin{array}{l}37 \text { pacientes } \\
\text { com AVC }\end{array}$ & - & $\begin{array}{l}\text { Teste Ullevaal } \\
\text { Aphasia } \\
\text { Screening } \\
\text { (UAS) }\end{array}$ & 5-15 minutos & $\begin{array}{l}\text { Expressão, } \\
\text { compreensão, } \\
\text { repetição, } \\
\text { leitura, } \\
\text { reprodução de } \\
\text { uma } \\
\text { sequência de } \\
\text { palavras, } \\
\text { escrita e } \\
\text { comunicação } \\
\text { livre }\end{array}$ \\
\hline $\begin{array}{l}\text { 14. Enderby \& } \\
\text { Crow (1996) }\end{array}$ & $\begin{array}{l}25 \text { pacientes } \\
\text { disfásicos pós } \\
\text { AVC }\end{array}$ & - & $\begin{array}{l}\text { Frenchay } \\
\text { Aphasia } \\
\text { Screening } \\
\text { Test (FAST) }\end{array}$ & 3-10 minutos & $\begin{array}{l}\text { Compreensão, } \\
\text { expressão, } \\
\text { leitura e } \\
\text { escrita }\end{array}$ \\
\hline $\begin{array}{l}\text { 15. Gibson, } \\
\text { MacLennan, } \\
\text { Gray \& Pentland } \\
(1991)^{37}\end{array}$ & $\begin{array}{l}6 \text { pacientes } \\
\text { com TCE e } 44 \\
\text { com AVC }\end{array}$ & - & $\begin{array}{l}\text { Frenchay } \\
\text { Aphasia } \\
\text { Screening } \\
\text { Test (FAST) }\end{array}$ & 3-10 minutos & $\begin{array}{l}\text { Compreensão, } \\
\text { expressão, } \\
\text { leitura e } \\
\text { escrita }\end{array}$ \\
\hline $\begin{array}{l}\text { 16. Mumby } \\
(1990)^{38}\end{array}$ & $\begin{array}{l}6 \text { pacientes } \\
\text { com AVC }\end{array}$ & - & $\begin{array}{l}\text { Aphasia } \\
\text { Screening } \\
\text { Test (AST) - } \\
\text { versão em } \\
\text { Panjabi }\end{array}$ & - & $\begin{array}{l}\text { Compreensão } \\
\text { de instruções } \\
\text { verbal e } \\
\text { escrita, } \\
\text { nomeação, } \\
\text { escrita, } \\
\text { cálculo, } \\
\text { repetição, } \\
\text { leitura, } \\
\text { descrição oral, } \\
\text { ditado, } \\
\text { habilidades } \\
\text { numéricas, } \\
\text { pareamento } \\
\text { de letras, } \\
\text { números, } \\
\text { palavras, } \\
\text { sentenças e } \\
\text { figuras }\end{array}$ \\
\hline $\begin{array}{l}\text { 17. O'Donnell, } \\
\text { Romero \& Leicht } \\
\text { (1990) }\end{array}$ & $\begin{array}{l}60 \\
\text { participantes } \\
\text { com } \\
\text { dificuldades } \\
\text { de } \\
\text { aprendizagem } \\
\text { e pacientes } \\
\text { com TCE }\end{array}$ & $\begin{array}{l}30 \\
\text { participantes } \\
\text { sem } \\
\text { dificuldade de } \\
\text { aprendizagem }\end{array}$ & $\begin{array}{l}\text { Retain-Indiana } \\
\text { Aphasia } \\
\text { Screening } \\
\text { Test (AST) }\end{array}$ & - & $\begin{array}{l}\text { Nomeação, } \\
\text { pronunciação } \\
\text { e } \\
\text { interpretação } \\
\text { de sentenças }\end{array}$ \\
\hline $\begin{array}{l}\text { 18. Enderby , } \\
\text { Wood, Wade \& } \\
\text { Hewer (1987) }\end{array}$ & $\begin{array}{l}50 \text { pacientes } \\
\text { com AVC }\end{array}$ & $\begin{array}{l}143 \\
\text { participantes } \\
\text { saudáveis }\end{array}$ & $\begin{array}{l}\text { Frenchay } \\
\text { Aphasia } \\
\text { Screening } \\
\text { Test (FAST) }\end{array}$ & 3-10 minutos & $\begin{array}{l}\text { Compreensão, } \\
\text { expressão, } \\
\text { leitura e } \\
\text { escrita }\end{array}$ \\
\hline
\end{tabular}


Quanto à segunda questão de pesquisa, "Quais as populações examinadas nesses estudos?", constatou-se que em 15 pesquisas a população alvo foi pacientes com AVC com sequelas linguísticas, seguida de dois estudos com pacientes com TCE, e apenas um com adultos saudáveis bilíngues. Esse resultado pode ser justificado pelo fato da afasia ser uma das consequências mais comuns e mais estudadas pós-AVC ${ }^{43}$. Atualmente estimase que $21-38 \%$ dos pacientes com AVC sejam afásicos ${ }^{44}$; além disso, sabe-se que a identificação e o diagnóstico precoce de déficits de linguagem representam um passo importante para maximizar os ganhos da reabilitação ${ }^{43}$.

Em resposta à terceira questão de pesquisa sobre quais são os componentes comunicativos mais avaliados por esses instrumentos, constatouse que a expressão foi avaliada em todos os testes, seguido da compreensão, que esteve presente em dez dos 11 testes analisados. A leitura e a escrita também foram avaliadas em praticamente todos os instrumentos. Esses dados eram previsíveis, visto que a população alvo predominante foi composta por pacientes com lesão em hemisfério esquerdo, tendo como dificuldades mais observadas déficits de expressão (como a anomia) e de compreensão da fala oral e escrita ${ }^{32}$. Pode-se constatar, ainda, que os componentes discursivo e pragmático, mais relacionados aos aspectos funcionais da linguagem ${ }^{45}$, não foram praticamente contemplados nos instrumentos que embasaram os estudos. Isto se deve provavelmente ao fato de que para seu exame demandam instruções e estímulos um pouco mais complexos do que avaliações de componentes tradicionais da linguagem em nível de som, palavra e sentença, necessitando, portanto, de maior tempo de aplicação.

Além das questões de pesquisa inicialmente previstas, outros aspectos observados por meio dessa revisão sistemática merecem ser brevemente discutidos. Dos 18 estudos analisados, oito tiveram como principal objetivo a validação de instrumentos. Parece haver uma maior preocupação com os parâmetros psicométricos na adaptação e construção de ferramentas de avaliação, o que se mostra muito promissor e benéfico para o avanço da área de avaliação da linguagem sob uma perspectiva neuropsicológica. A obtenção de evidências de validade, fidedignidade e dados normativos enriquecem a qualidade de ferramentas avaliativas clínica e teoricamente embasadas ${ }^{46}$. Em complementaridade, sete dos artigos analisados mencionaram a aplicação do $F A S T^{23-25,31,36,37,40}$ e dois do $M A S T^{27,33}$, sendo que o FAST foi adaptado para um idioma não ocidental (Panjabi) ${ }^{38}$. Os testes FAST e MAST têm como objetivo identificar pacientes afásicos no quadro agudo ${ }^{43}$. São descritos como sendo de rápida e de fácil aplicação e podem ser administrados por qualquer profissional experiente da área da saúde. O FAST é considerado um instrumento sensível; no entanto, sua especificidade pode ser afetada pela presença de déficits perceptivos, como heminegligência visual, por desatenção, analfabetismo, surdez e confusão mental. O MAST também contém alguns itens que requerem busca visual dos estímulos apresentados e os resultados podem ser negativamente afetados na presença de um déficit nesse processamento. No entanto, salienta-se que é dificilmente um instrumento ou tarefa/subteste poderá avaliar apenas um determinado componente cognitivo, por exemplo, um processo linguístico, uma vez que a cognição humana é tão complexa que no mínimo atenção e percepção auditiva ou visual estarão envolvidas para o input da informação a ser processada linguisticamente. Deste modo, é conveniente incluir outros instrumentos que avaliem predominantemente estas funções na bateria de avaliação dos pacientes para que se possa promover uma análise intertestes identificando-se quais são os componentes primária e secundariamente comprometidos.

\section{CONCLUSÃO}

As baterias de avaliação da comunicação têm por finalidade analisar componentes comunicativos com o intuito de alcançar um diagnóstico funcional e, consequentemente, auxiliar no prognóstico e no processo de reabilitação. Isso porque os instrumentos utilizados na prática clínica são longos e, muitas vezes, não podem ser usados na íntegra em hospitais ou no sistema público de saúde em que o tempo disponível para testagem é mais reduzido que em outros contextos.

Essa revisão apresentou um panorama dos instrumentos mais utilizados, descrevendo suas populações clínicas e componentes de linguagem avaliados. Nessa busca nenhum instrumento brasileiro foi encontrado revelando a escassez desse tipo de instrumento no contexto nacional. Dessa forma, verifica-se a necessidade de estudos futuros para suprir essa lacuna. Mais especificamente, até mesmo em nível internacional, parece haver uma demanda ainda não atendida de ferramentas clinicas de avaliação breve de componentes funcionais da linguagem, como pragmático-inferencial e discursivo. Tais componentes por sua complexidade e estreita relação com outros componentes cognitivos, principalmente mnemônicos e executivos, tendem a estar afetados em diversos quadros neurológicos e psiquiátricos, requerendo uma avaliação acurada. 


\section{ABSTRACT}

Background: the brief assessment of communication is a useful resource to health professionals. It provides information on the functional diagnosis, planning and execution of expanded evaluations and rehabilitation programs to be undertaken in each case. Purpose: to submit a systematic review on the use of standardized instruments regarding brief assessment of communication, and characterize the target population. Conclusion: based on the conducted research, 18 manuscripts with 10 brief instruments were found. The most examined population was post-stroke patients. Expression, comprehension, reading and writing were the most prevalent linguistic components in the evaluations.

KEYWORDS: Evaluation; Language; Communication

\section{REFERÊNCIAS}

1. Haan EHF, Zandvoort MJE, Nys GMS, Kappelle LJ. Neuropsychological sequelea of cerebellar stroke. J Neurol Scien. 2009; 283: 269.

2. Dikmen SS, Corrigan JD, Levin HS, Machamer J, Stiers W, Weisskopf MG. Cognitive outcome following traumatic brain injury. J Head Trauma Rehabil. 2009; 24: 430-8.

3. Mansur LL, Carthery MT, Caramelli P, Nitrini R. Linguagem e cognição da doença de Alzheimer. Psicol: Reflexão e Crítica. 2005; 18(3): 300-7.

4. Helm-Estabrooks N. Aphasia Diagnostic Profiles (ADP). Dallas: PRO-ED; 1992.

5. Goodglass H, Kaplan E. Assessment of aphasia and related disorders. Philadelphia: Lea \& Febiger; 1972.

6. Goodglass H, Kaplan E, Barresi B. Boston Diagnostic Aphasia Examination. 3th ed. Texas: The Psychological Corporation; 2000.

7. Kaplan E, Goodglass H, Weintraub S. The Boston Naming Test. Philadelphia: Lippincott Williams \& Wilkins; 2001.

8. Benton L, Hamsher S, Sivan A. Multilingual Aphasia Examination (MAE-E). Journal of the International Neuropsychological Society. Cambridge University Press; 1994.

9. Spreen O, Benton AL. Neurosensory Center Comprehensive Examination for Aphasia: Manual of instructions (NCCEA). Victoria: University of Victoria; 1977.

10. Porch B. Porch Index of Comunicative Ability (PICA). Palo Alto: Consulting Psychologists Press; 1981.

11. Lapointe L, Horner J. Reading Comprehension Battery for Aphasia (RCBA-2). Dallas: PRO-ED; 1998.

12. De Renzi EY, Yignolo L. The token test: a sensitive test to detect receptive disturbances in aphasics. Brain. 1962; 85: 665-78.
13. Kertesz A. Western Aphasia Battery. New York: Grune \& Stratton; 1982.

14. Ostrosky-Solís F, Ardila A, Rosselli M. NEUROPSI: A brief neuropsychological test battery in Spanish with norms by age and educational level. J Int Neuropsychol Soc. 1999; 5: 413-33.

15. Vitiello APP, Ciríaco JGM, Takahashi DY, Nitrini R, Caramelli P. Avaliação cognitiva breve de pacientes atendidos em ambulatório de neurologia geral. Arq Neuropsiquiatr. 2007; 65(2-A):299-303.

16. Reys BN, Bezerra AB, Vilela ALS, Keusen AL, Marinho V, Paula E, Laks J. Diagnóstico de demência, depressão e psicose em idosos por avaliação cognitiva breve. Rev Assoc Med Bras. 2006; 52: 401-4.

17. Fonseca RP, Salles JF, Parente MAMP. Instrumento de Avaliação Neuropsicológica Breve NEUPSILIN. São Paulo: Vetor; 2009.

18. Yates DB, Trentini CM, Tosi SD, Corrêa SK, Poggere LC, Valli F. Apresentação da Escala de Inteligência Wechsler abreviada (WASI). Aval. Psicol. 2006; 5(2): 227-33.

19. Beato RG, Nitrini R, Formigoni AP, Caramelli P. Brazilian version of the frontal assessment battery (FAB): preliminary data on administration to healthy elderly. Dement \& Neuropsychol. 2007; 1: 59-65.

20. Nelson HE. A modified card sorting test sensitive to frontal lobe defects. Cortex. 1976; 12: 313-24.

21. Fonseca RP, Parente MAMP. Metanálise de estudos do processamento comunicativo em indivíduos com lesão vascular direita. Est Psico (Campinas). 2007; 24(4):529-38.

22. Brookshire $\mathrm{RH}$. Introduction to neurogenic communication disorders. Missouri: Mosby; 2003.

23. Hilari K, Northcott S, Roy P, Marshall J, Wiggins RD, Chataway J, Ames D. Psychological distress after stroke and aphasia: the first six months. Clin Rehabil. 2010; 24:181-90.

24. Hilari K, Lamping DL, Smith SC, Northcott S, Lamb A, Marshall J. Psychometric properties of the Stroke and Aphasia Quality of Life Scale 
(SAQOL-39) in a generic stroke population. Clini Rehabil. 2009; 23: 544-57.

25. Long A, Hesketh A, Bowen A. Communication outcome after stroke: a new measure of the carer's perspective. Clin Rehabil. 2009; 23: 846-56.

26. Casas R, Calamia M, Tranel D. A screening test of English naming ability in bilingual Spanish/ English speakers. J Clinic Exp Neuropsychol. 2008; 30(8): 956-66.

27. Kostalova M, Bartkova E, Sajgali K, Dolenska A, Dusek L, Bednarik J. A standardization study of the Czech version of the Mississippi Aphasia Screening Test (MASTcz) in stroke patients and control subjects. Brain Injury. 2008; 22(10): 793-801.

28. Laska AC, Kahan T, Hellblom A, Murray V, von Arbin M. Design and methods of a randomized controlled trial on early speech and language therapy in patients with acute stroke and aphasia. Top Stroke Rehabil. 2008; 15(3): 256-61.

29. Sabe L, Courtis MJ, Saavedra MM, Prodan V, Luján-Calcagno M, Melián S. Desarrollo y validación de una batería corta de evaluación de la afasia: 'bedside de lenguaje'. Utilización en un centro de rehabilitación. Rev Neurol. 2008; 46(8):454-60.

30. Theodoros D, Hill A, Path BS, Russell T, Ward E, Wootton R. Assessing acquired language disorders in adults via the internet. Telemed e ehealth. 2008; 14(6): 552-9.

31. Edwards DF, Hahn MG, Baum CM, Perlmutter MS, Sheedy C, Dromerick AW. Screening patients with stroke for rehabilitation needs: validation of the post-stroke rehabilitation guidelines. Neurorehabil Neural Repair. 2006; 20(1): 42-8.

32. Kalbe K, Reinhold N, Brand M, Markowitsch $\mathrm{HJ}$, Kessler J. A new test battery to assess aphasic disturbances and associated cognitive dysfunctions - german normative data on the aphasia check list. J Clin Exp Neuropsychol. 2005; 27: 779-94.

33. Nakase-Thompson R, Manning E, Sherer M, Yablon SA, Gontkovsky SLT, Vickery C. Brief assessment of severe language impairments: Initial validation of the Mississippi aphasia screening test. Brain Injury. 2005; 19(9): 685-91.

http://dx.doi.org/10.1590/S1516-18462011005000031 RECEBIDO EM: 30/09/2010

ACEITO EM: 07/02/2011

Endereço para correspondência:

Fabíola Schwengber Casarin

Rua Pedro Ivo, 378 apto 601

Porto Alegre, RS

CEP: 90450-210

E-mail: fabiolacasarin @gmail.com
34. Blake H, McKinney M, Treece K, Lee E, Linconl NB. An evaluation of screening measures for cognitive impairment after stroke. Age Ageing. 2002; 31: 451-6.

35. Thommessen B, Thoresen GE, Bautz-Holter E, Laake K. Screening by nurses for aphasia in stroke - the Ullevaal Aphasia Screening (UAS) test. Disabil Rehabil. 1999; 21(3): 110-5.

36. Enderby P, Crow E. Frenchay Aphasia Screening Test: validity and comparability. Disabil Rehabil. 1996; 18(5): 238-40.

37. Gibson L, MacLennan WJ, Gray C, Pentland B. Evaluation of a comprehensive assessment battery for stroke patients. Int J Rehabil Res. 1991; 14(2):93-100.

38. Mumby, K. Preliminary results from using the Panjabi adaptation of the Aphasia Screening Test. Br J Disord Commun. 1990; 25(2): 209-26.

39. O'Donnell JP, Romero JJ, Leicht D. A comparison of language deficits in learning-disabled, had-injured, and nondisabled young adults. J Clin Psychol. 1990; 46(3):310-5.

40. Enderby PM., Wood VA, Wade DT, Hewer RL. The Frenchay Aphasia Screening Test: A short, simple test for aphasia appropriate for nonspecialists. Inter J Rehabil Med. 1987; 8: 166-70.

41. Horton Jr. AM, Horton III AM. Brief and comprehensive neuropsychological assessment of alcohol and drug abuse. In D'Amato RC, Hartlage LC, editors. Essentials of neuropsychological assessment - treatment plannig for rehabilitation. New York: Springer Publishing Company; 2008. p. 293-314.

42. William JM. Brief and extended neuropsychological assessment of aging and dementia. In D'Amato RC, Hartlage LC, editors. Essentials of neuropsychological assessment treatment plannig for rehabilitation. New York: Springer Publishing Company; 2008. p. 315-32.

43. Salter K, Jutai J, Foley N, Hellings C, Teasell R. Identification of aphasia post-stroke: A review of screening assessment tools. Brain Inj. 2006; 20(6): 559-68.

44. Berthier ML. Poststroke aphasia : epidemiology, pathophysiology and treatment. Drugs Aging. 2005; 22(2): 163-82.

45. Dennis $M$. Language disorders in children with central nervous system injury. J Clin Exper Neuropsychol. 2010; 32(4): 417-32.

46. Pawlowski J, Fonseca RP, Salles JF, Parente MAMP, Bandeira DR. (2008). Evidências de validade do Instrumento de Avaliação Neuropsicológica Breve NEUPSILIN. Arq Brasil Psicol. 2003; 60: 101-16. 